\title{
Selection of a reference gene for studies of adipose tissues of toothed whales
}

\author{
Jayan Senevirathna $^{1}$, Ryo Yonezawa ${ }^{1}$, Taiki Saka ${ }^{1}$, Yoji Igarashi ${ }^{2}$, Kazutoshi Yoshitake $^{1}$, \\ Shigeharu Kinoshita ${ }^{1}$, Noriko Funasaka ${ }^{2}$, and Shuichi Asakawa ${ }^{1}$ \\ ${ }^{1}$ The University of Tokyo - Hongo Campus \\ ${ }^{2}$ Mie University
}

April 28, 2021

\begin{abstract}
Fat metabolism in toothed whales is different from other mammals. RT-qPCR is still a reliable technique for studying the relative expressions of various genes involved in metabolism. This study was done for Risso's dolphin, a toothed whale and information produced here will be important for further transcriptomics studies focused on unrevealed marine mammal fat metabolism. In this study, we sought to identify a suitable reference gene with minimum resources. Seven candidate reference genes ZC3H10, FTL, LGALS1, RPL27A, GAPDH, FTH1 and DCN were initially tested for amplification efficiency using RTqPCR by producing standard curves. Then, three nearly $100 \%$ efficient genes FTL, LGALS1 and GAPDH were selected for the gene stability analysis to determine one stable gene across eight different fat tissues, liver, and muscle of Risso's dolphins based on four algorithms, provided in geNorm, NormFinder, BestKeeper and Delta Ct. Finally, a RefFinder comprehensive ranking was done based on stability values and the genes were ranked as: FTL>LGALS1>GAPDH. The FTL and LGHLS were identified as the most stable genes; however, GAPDH was third, a well-known housekeeping gene for mammals. Finally, we suggest using FTL as a reliable reference gene for functional genomics studies on toothed whales in the future.
\end{abstract}

\section{Hosted file}

EE_jdms_21-4-23.pdf available at https://authorea.com/users/410521/articles/519871-selectionof-a-reference-gene-for-studies-of-adipose-tissues-of-toothed-whales 